\title{
Diagnostic Yield of Serial Sputum Smear Microscopy Examination at Different Institutional Settings
}

Daniel Melese Desalegn ${ }^{1-3^{*}}$

${ }^{1}$ Ethiopian Public Health Institute (EPHI), Addis Ababa, Ethiopia

${ }^{2}$ Addis Ababa Public Health Research and Emergency Management Core Process, Addis Ababa City Administration Health Bureau, Addis Ababa, Ethiopia

${ }^{3}$ College of Health Sciences, School of Clinical Laboratory Science, Addis Ababa University, Addis Ababa, Ethiopia

*Corresponding author: Daniel Melese Desalegn, Ethiopian Public Health Institute (EPHI), Addis Ababa, Ethiopia, Tel: +251911934843; E-mail: danimelese2013@gmail.com

Received date: November 13, 2018; Accepted date: November 30, 2018; Published date: December 08, 2018

Copyright: (c) 2018 Desalegn DM. This is an open-access article distributed under the terms of the Creative Commons Attribution License; which permits unrestricted use; distribution; and reproduction in any medium; provided the original author and source are credited.

\begin{abstract}
Introduction: Number of samples and time of sputum collection has a great impact on the positivity yield of sputum smear microscopy examination. However, the impact is not yet extensively studied in Ethiopia. Therefore, the aim of this study was to assess the serials sputum sample collection impacts on the diagnostic yield of smear microscopy examination in Ethiopia at variation at different institutional settings.
\end{abstract}

Methods: Institution based retrospective cohort study was conducted from October 2011 to March 2016. Two thousand four hundred sixty-three sputum smear microscopy positive result were reviewed from TB patient records. The collected data were computerized using Epi-info software and analyzed using SPSS version 20 software. Numerical summaries were used to describe the positivity yield of spot, morning and spot (SMS) sputum smear microscopy examination.

Results: Of 2463 sputum smear-positive TB cases, 2384 (96.8\%), 2458 (99.8\%) and 2394 (97.2\%) were detected by the first spot, morning and second spot sputum smear microscopy diagnosis, respectively. On the other hand, morning sputum smear results had the incremental yield of $74(3.0 \%)$ and $64(2.6 \%)$ from first spot sample and second spot sample, respectively. The results of $1892(76.8 \%)$ TB cases were in agreement (detected) by three consecutive sputum specimen (SMS).

Conclusion: The three consecutive sputum smear microscopy examination had $3 \%$ incremental yield compare to spot-spot (SS). The hospitals were participating in International External Quality Assurance Schemes (IEQAS) had better incremental (increased) yield in both SMS and SS approach compared to the health centers. Hence, strong TB quality assurances systems should be implemented in respective health facilities before practicing two spots (SS) sputum smear microscopy diagnostic approach as routine to TB laboratory diagnostic method.

Keywords: Sputum smear; Microscopy diagnostic; Serial sputum; Addis Ababa; Ethiopia

Abbreviations: AFB: Acid-Fast Bacillus (or Bacilli); EQA: External Quality Assessment; IEQAS: International External Quality Assurance Schemes; REQAS: Regional External Quality Assurance Schemes; IQC: Internal Quality control; SMS: Spot-Morning-Spot; TB: Tuberculosis; PTB: Pulmonary Tuberculosis; WHO: World Health Organization; ZN: Ziehl-Neelsen Stain

\section{Introduction}

Tuberculosis (TB) remains one of a serious public health threat of the world. An appropriate laboratory diagnostic procedure plays an important role in TB prevention. Sputum smear microscopy is one of the key pillars of strategies for TB control [1,2]. However, it has a significant limitation in its performance. For instance, its sensitivity is grossly compromised when the bacterial load is less than 10,000 organisms per ml sputum sample [3]. Its positivity also depends on the number of samples and time of sputum collection [1-4]. Hence the diagnostic yield of sputum smear microscopy examination increases in the 2-days Spot-Morning-Spot (SMS) diagnostic approach compares to the same day Spot-Spot (SS) $[3,5]$.

Although SMS increase the positivity yielding of sputum smears microscopy, it is inconvenient for patients, who have to make multiple visits to health facilities to submit multiple (SMS) sputum specimens over several days. As a result, some patients who do not come back for repeated sputum examination become diagnostic defaulter [6]. To reduce patient loss in the diagnostic pathway (defaulter rate), World Health Organization (WHO) has recently recommended a reduction in the number of specimens examined from three (SMS) to two (SS) in settings where appropriate external microscopy quality assurance systems are in place [6]. In addition, the SS diagnostic approach may allow patients to start anti-TB treatment on the same day, which would contribute to lowering patient-related costs [6].

Studies show that SS can detect the vast majority (95\%-98\%) of smear-positive TB patients. However, this might not be true in all setting, moreover, we are not aware of any published study in Addis Ababa targeting the diagnostic yield of spot and morning sputum samples. Therefore, this study was designed to assess the diagnostic 
Page 2 of 4

yield of serials sputum smear microscopy examination at different institutional settings $[7,8]$.

\section{Materials and Methods}

\section{Study design and setting}

Public health institution based retrospective cohort study was conducted in Addis Ababa, the capital city of Ethiopia. The study was conducted in a Public health institution. Health institutions were selected using simple random sampling with the plan of obtaining three hospitals from six public hospitals and ten health centers (one from each sub-city). The health facilities' sputum smear microscopy records, from a period of 2011-2016 were used as data source. Individual patients' data documented in the TB record book of these laboratories were used as study subjects. TB laboratory quality assurances system was assessed by using a structured questionnaire and observational checklists.

\section{Data management and statistical analysis}

Before extracting data from records, data collectors were adequately trained. Quality of data collection process was supervised and monitored by the principal investigator. Two thousand four hundred sixty-three sputum smear microscopy positive result were collected from TB patient records. The collected data were checked for completeness and consistency and computerized using Epi-info software. The data were coded and analyzed using SPSS version 20.0 (SPSS Inc. Chicago, USA) software. Descriptive numerical summaries were used to describe the findings.

\section{Operational definition}

Bacillary-load yields in the serial sputum samples as categorized: actual numbers $=1-9$ Acid Fast Bacilli (AFB) per 100 fields, $+1=10-99$ AFB per 100 fields, $+2=1-10$ AFB per field, checked 50 fields and $+3=$ more than $10 \mathrm{AFB}$ per field, checked 20 fields.

\section{Result}

In this study, a total of 2463 sputum smear-positive TB patients' records were reviewed. Among these TB patients who were registered in the study health facilities, 1091 (44.3\%) were in three public hospitals and the remaining $1372(55.7 \%)$ were in ten health centers. Of 2463 TB cases, 1291 (52.4\%) were males, 70 (2.8\%) were below the age of 15 years and 2393 (97.2\%) were above 19 years. Records were incomplete (not documented) the age of $194(7.9 \%)$ and the sex of 95 (3.9\%) patients (Table 1 ).

\begin{tabular}{|c|c|c|}
\hline Variables & Number & Percent \\
\hline \multicolumn{3}{|c|}{ Age group in year } \\
\hline$<15$ & 70 & 2.8 \\
\hline $15-18$ & 170 & 6.9 \\
\hline $19-35$ & 1435 & 58.3 \\
\hline $36-55$ & 456 & 18.5 \\
\hline$>55$ & 138 & 5.6 \\
\hline Data missing & 194 & 7.9 \\
\hline
\end{tabular}

\begin{tabular}{|c|c|c|}
\hline Total & 2463 & 100 \\
\hline \multicolumn{3}{|c|}{ Sex of TB patients } \\
\hline Male & 1291 & 52.4 \\
\hline Female & 1077 & 43.7 \\
\hline Data missing & 95 & 3.9 \\
\hline Total & 2463 & 100 \\
\hline
\end{tabular}

Table 1: Distribution of pulmonary positive tuberculosis patients by age group and sex, at public health facilities, from 2011-2016 $(n=2463)$.

Of 2463 sputum smear-positive TB cases, 2384 (96.8\%), 2458 (99.8\%) and 2394 (97.2\%) were detected by the first spot; morning and second spot sputum smear microscopy diagnosis respectively. Yield variation of morning sputum smear results had the incremental yield of $74(3.0 \%)$ and $64(2.6 \%)$ from first spot sample and second spot sample respectively. The results of $1892(76.8 \%)$ TB cases were in agreement (detected) by three consecutive sputum specimen (SMS). Comparing the diagnostic yielding variation at different institutional settings, the morning sputum smear positive result had the incremental yield of $29(2.6 \%)$ in hospitals and $47(1.9 \%)$ in the health centre. The first spot smear microscopy missed 32 (2.9\%) TB cases in hospitals and $47(3.4 \%)$ in health centres, while the second smear microscopy missed $25(2.3 \%)$ TB cases in hospitals and $43(3.1 \%)$ in health centres (Table 2).

\begin{tabular}{|c|c|c|c|c|}
\hline \multicolumn{2}{|l|}{ Variables } & \multirow{2}{*}{$\begin{array}{l}\text { Positive } \\
\text { Number (\%) } \\
1059 \text { (97.1) }\end{array}$} & \multirow{2}{*}{$\begin{array}{l}\text { Negative } \\
\text { Number (\%) } \\
32(2.9)\end{array}$} & \multirow{2}{*}{$\begin{array}{l}\text { Total } \\
\text { Number (\%) } \\
1091(44.3)\end{array}$} \\
\hline First spot & Hospitals & & & \\
\hline & Health centres & 1325 (96.6) & $47(3.4)$ & 1372 (55.7) \\
\hline & Total & $2384(96.8)$ & $79(3.2)$ & $2463(100)$ \\
\hline \multirow[t]{3}{*}{ Morning } & Hospitals & 1088 (99.7) & $3(0.3)$ & $1091(44.3)$ \\
\hline & Health centres & $1370(99.1)$ & $2(0.1)$ & $1372(55.7)$ \\
\hline & Total & 2458 (99.8) & $5(0.2)$ & $2463(100)$ \\
\hline \multirow[t]{3}{*}{ Second spot } & Hospitals & 1066 (97.7) & $25(2.3)$ & $1091(44.3)$ \\
\hline & Health centres & 1329 (96.9) & $43(3.1)$ & 1372 (55.7) \\
\hline & Total & 2395 (97.2) & $68(2.8)$ & $2463(100)$ \\
\hline
\end{tabular}

Table 2: Diagnostic yielding variation among serials of sputum smears microscopy at public health facilities in Addis Ababa, Ethiopia.

Regarding bacillary-load yields in the serial sputum samples, out of 2384 first spot sputum smear-positive cases, the bacillary-load of 189 (7.9\%) were actual numbers (1-9 bacilli in 100 fields), 535 (22.4\%) were +1 (10-99 bacillus in 100 fields), 378 (15.9\%) were +2 (1-10 bacillus per field, checked 50 fields) and 325 (13.6\%) were +3 (more than 10 bacilli per field, checked 20 fields). The bacillary-load of the remaining 957 (40.1\%) first spot sputum smear results were not recorded on the log book (Reported by non-grading systems). From the total 2458 morning sputum smear-positive cases, the bacillary-load of $188(7.6 \%)$ were actual numbers, $492(20.0 \%)$ were $+1,311(12.7 \%)$ were +2 and $251(10.2 \%)$ were +3 . The bacillary-load of the remaining $1216(49.5 \%)$ morning sputum smear results were not recorded on the log book 
Page 3 of 4

(Reported by non-grading systems). Of 2394 second spot sputum smear-positive cases, the bacillary load of $156(6.5 \%)$ were actual numbers, $423(17.7 \%)$ were $+1,431(18.0 \%)$ were +2 and $412(17.2 \%)$ were +3 . The bacillary-load of the remaining $972(40.6 \%)$ second spot sputum smear results were not recorded on the log book (Table 3).

\begin{tabular}{|c|c|c|c|c|c|c|}
\hline $\begin{array}{l}\text { Sample } \\
\text { s }\end{array}$ & $\begin{array}{l}\begin{array}{l}\text { Actual } \\
\text { numbers }\end{array} \\
1-9 / 100 \\
\text { Number } \\
(\%)\end{array}$ & $\begin{array}{l}1 \\
\text { Number } \\
(\%)\end{array}$ & $\begin{array}{l}2 \\
\text { Number } \\
(\%)\end{array}$ & $\begin{array}{l}3 \\
\text { Number } \\
(\%)\end{array}$ & $\begin{array}{l}\text { Data } \\
\text { missing } \\
\text { (no } \\
\text { grade) }\end{array}$ & $\begin{array}{l}\text { Total } \\
\text { Number } \\
(\%)\end{array}$ \\
\hline Spot 1 & $189(7.9)$ & $\begin{array}{l}535 \\
(22.4)\end{array}$ & $\begin{array}{l}378 \\
(15.9)\end{array}$ & $\begin{array}{l}325 \\
(13.6)\end{array}$ & $\begin{array}{l}957 \\
(40.1)\end{array}$ & $\begin{array}{l}2384 \\
(100.0)\end{array}$ \\
\hline Morning & $\begin{array}{l}188 \\
(7.6)\end{array}$ & $\begin{array}{l}492 \\
(20.0)\end{array}$ & $\begin{array}{l}311 \\
(12.6)\end{array}$ & $\begin{array}{l}251 \\
(10.2)\end{array}$ & $\begin{array}{l}1216 \\
(49.5)\end{array}$ & $\begin{array}{l}2458 \\
(100.0)\end{array}$ \\
\hline Spot 2 & $156(6.5)$ & $\begin{array}{l}423 \\
(17.7)\end{array}$ & $\begin{array}{l}431 \\
(18.0)\end{array}$ & $\begin{array}{l}412 \\
(17.2)\end{array}$ & $\begin{array}{l}972 \\
(40.6)\end{array}$ & $\begin{array}{l}2394 \\
(100.0)\end{array}$ \\
\hline
\end{tabular}

Table 3: Bacillary load among serials of sputum smears microscopy at public health facilities in Addis Ababa, Ethiopia.

\section{TB laboratory quality assurances}

Out of the 13 visited laboratories, all laboratories were participated in Regional External Quality Assurance Schemes (REQAS) on ZNstained sputum smear microscopic slide rechecking. However, only three hospital laboratories have participated in International External Quality Assurance Schemes (IEQA). Out of the 13 laboratories, 4 $(30.8 \%)$ reported the false reading of sputum smear microscopic result in previous (had misreading practice). There was a lack of internal quality control materials in all assessed laboratories. However, 8 (61.5\%) laboratories prepare smears from known positive and negative sputum as internal quality control. About 87 (53.8) laboratories were run IQC to check new batch reagents quality before staining patient sample. All visited laboratories were checked sputum quality before processing and all had a standard laboratory request form, result log book and standard operating procedures (SOPs). All laboratories staff members have trained on sputum smears microscopy diagnosis (Table $4)$.

\begin{tabular}{|l|l|l|l|}
\hline \multicolumn{2}{|l|}{ Variables } & Number & Percent \\
\hline Laboratory participation in IEQAS & Yes & 3 & 23.1 \\
\cline { 2 - 4 } & No & 10 & 76.9 \\
\hline \multirow{2}{*}{ Run IQC slides parallel with routine ZN staining } & Yes & 8 & 61.5 \\
\cline { 2 - 5 } & No & 5 & 38.5 \\
\hline Having SOPs, guideline and manuals & Yes & 13 & 100 \\
\cline { 2 - 5 } & No & 0 & 0 \\
\hline Laboratory participation in REQAS & Yes & 13 & 100 \\
\cline { 2 - 5 } & No & 0 & 0 \\
\hline Having IQC materials & Yes & 2 & 15.4 \\
\cline { 2 - 5 } & No & 11 & 84.6 \\
\hline Having standard TB result registration log-book & Yes & 13 & 100 \\
\cline { 2 - 5 } & No & 0 & 0 \\
\hline
\end{tabular}

\begin{tabular}{|l|l|l|l|}
\hline Checking the quality of sputum & Yes & 13 & 100 \\
\cline { 2 - 4 } & No & 0 & 0 \\
\hline Having a false positive & Yes & 4 & 30.8 \\
\cline { 2 - 4 } & No & 9 & 69.2 \\
\hline $\begin{array}{l}\text { Staff received training on ZN sputum smears } \\
\text { microscopy }\end{array}$ & Yes & 13 & 100 \\
\cline { 2 - 4 } & No & 0 & 0 \\
\hline Reagent verification & Yes & 7 & 53.8 \\
\cline { 2 - 4 } & No & 6 & 44.2 \\
\hline
\end{tabular}

Table 4: TB laboratory quality assurances assessment in Addis Ababa, Ethiopia.

\section{Discussion}

In this study, we reviewed $2463 \mathrm{~TB}$ patients' laboratory records to determine the diagnostic yield of serials sputum smear microscopy. Of the 2463 sputum smear-positive TB cases, $96.8 \%$ were positive on the first spot and $97.2 \%$ were positive on second spot sputum smear microscopy, which was comparable to $95-98 \%$ reported in WHO same day sputum microscopy policy statement [6]. However, it was higher than the study conducted in South Western Uganda which reported $95.0 \%$ detected by first spot samples and $92.5 \%$ detected by second spot samples [9]. In the present study, the agreement of the three consecutive sputum smear microscopy (SMS) result was $76.8 \%$, which was lower than $82.0 \%$ reported India [10]. Sputum smear microscopy reading is highly dependent on the training and diligence of microscopist, availability of supplies, equipment and time taken for sputum smear microscopy reading. In addition, health-system-related factors including poor TB laboratory quality assurances systems and laboratory infrastructures might have impacts on TB case detection by using sputum smear microscopy.

In the current study, near to $3 \%$ of morning sputum microscopy results had incremental yields from the two spot (SS) sample, which was higher than $0.0 \%$ (no) incremental yields reported in South Western Uganda, $0.29 \%$ reported in India and $0.37 \%$ reported Rajahmundry India $[9,11,12]$. The incremental yield difference among serials of sputum samples might be related to many factors including: Instruction given to patients to submit quality and adequacy of sputum samples, time of sputum collection (the bacillary load increase in sputum spout out in the early morning), patient understanding how to collect quality of sputum samples and professional exercise on the way to guide and explain the quality of sputum submit for diagnosis and testing performed on good quality of sputum may impacts on yield of sputum microscopy diagnostic.

In this study, spot sputum smear microscopy failed to detect TB cases were higher in the health centre compared to the hospital. This might be due to hospitals' have well-equipped than health centres' laboratories. On the contrary, poor TB laboratory quality assurance systems including overcrowded and poorly ventilated laboratory spaces might be contributed to the low detection of TB in health centres. In our study setting none of the health centre laboratories were participating in IEQS, which indicates that the successful implementation of TB microscopy quality assurance systems might be expected to improve the detection level of the SS approach. 
Citation: Desalegn DM (2018) Diagnostic Yield of Serial Sputum Smear Microscopy Examination at Different Institutional Settings. J Trop Dis 7: 295. doi:10.4172/2329-891X.1000294

Page 4 of 4

\section{Conclusion and Recommendations}

The present study shows that SMS sputum smear microscopy examination had $3 \%$ incremental yield compare to SS. The hospitals were participating in International External Quality Assurance Schemes (IEQA) scheme had a better incremental yield in both SMS and SS approach compare to the health centers. Hence, strong TB quality assurances systems should be implemented in respective health facilities before practices two spots (SS) sputum smear microscopy diagnostic approach as routine to TB laboratory diagnostic method.

\section{Consent to Participate}

Not applicable

\section{Consent to Publish}

Before the research work, permission was obtained from Addis Ababa City Administration Health Bureau Public Health Research and Emergency Management Core Processes.

\section{Availability of Data and Materials}

All data were presented in the result parts of the manuscript.

\section{Competing Interest}

The author(s) declare that they have no competing interest.

\section{Author Contributions}

DMD designed the study, develops the proposal, lead data collection, analysis and writing up of the result. AA and ATB critically review and made progressive suggestions from the design to final write up of the result. All of the authors have participated in data collection, analysis, interpretation, critically evaluated and approved the manuscript.

\section{Acknowledgments}

The authors would like to thank Addis Ababa City administration health bureau and respective health facilities for their cooperation. Our thanks also extend to data collectors and supervisors for their cooperation during the study period.

\section{References}

1. World Health Organization (2014) Global tuberculosis report 2014.

2. Rao S (2009) Sputum smear microscopy in DOTS: Are three samples necessary? An analysis and its implications in tuberculosis control. PMC Lung India 26: 1-3.

3. WHO (2007) Proposed reduction of a number of smears for the diagnosis of Pulmonary TB. Background document. Geneva.

4. Warren JR, Bhattacharya M, De Almeida KNF, Trakas K, Peterson LR (2000) A minimum $5.0 \mathrm{ml}$ of sputum improves the sensitivity of acid-fast smear for Mycobacterium tuberculosis. Am J Respir Crit Care Med 161: 1559-1562.

5. Sakundarno M, Nurjazuli N, Jati SP, Sariningdyah R, Purwadi S, et al. (2009) Insufficient quality of sputum submitted for tuberculosis diagnosis and associated factors in Klaten district, Indonesia. BMC Pulm Med. 9: 16.

6. World Health Organization (2011) Same-day diagnosis of tuberculosis by microscopy. Policy statement.

7. Siddiqi K, Lambert M-L, Walley J (2003) Clinical diagnosis of smearnegative pulmonary tuberculosis in low-income countries: the current evidence. Lancet Infect Dis 3: 288-296.

8. Apers L, Mutsvangwa J, Magwenzi J, Chigara N, Butterworth A, et al (2003) A comparison of direct microscopy, the concentration method and the mycobacteria growth indicator tube for the examination of sputum for acid-fast bacilli. Int J Tubercul Lung Dis 7: 376-381.

9. Bazira J, Mwambi B, Muyindike W (2015) Feasibility for Same day tuberculosis diagnosis using the smear microscopy approach in rural South Western Uganda. Adv Res 4: 151-155.

10. Nayak P, Kumar AMV, Claassens M, Enarson DA, Satyanarayana S, et al. (2013) Comparing same day sputum microscopy with conventional sputum microscopy for the diagnosis of Tuberculosis-Chhattisgarh, India. PLoS ONE 8: e74964.

11. Chandra TJ (2012) Same day sputum smear microscopy approach for the diagnosis of pulmonary tuberculosis in a microscopy centre at Rajahmundry. Indian J Tuberc 59: 141-144.

12. Chandra TJ, Raj RS, Sharma YV (2014) Same day sputum smear microscopy approach with modified $\mathrm{ZN}$ staining for the diagnosis of pulmonary tuberculosis in a microscopy centre at Rajahmundry. Indian J Med Microbiol 32: 153-156. 